EMBRYARIDDLE
Aeronautical University

SCHOLARLY COMMONS

\section{International Journal of Aviation,} Aeronautics, and Aerospace

\title{
Damage characterization of aircraft fuselage using vibrothermography technique-review and analysis
}

\author{
Abbasali Saboktakin \\ University of sistan and baluchestan, alaptakin@gmail.com
}

Follow this and additional works at: https://commons.erau.edu/ijaaa

Part of the Biomedical Engineering and Bioengineering Commons, Manufacturing Commons, and the Other Aerospace Engineering Commons

\section{Scholarly Commons Citation}

Saboktakin, A. (2018). Damage characterization of aircraft fuselage using vibrothermography techniquereview and analysis. International Journal of Aviation, Aeronautics, and Aerospace, 5(5). https://doi.org/ 10.15394/ijaaa.2018.1300

This Article is brought to you for free and open access by the Journals at Scholarly Commons. It has been accepted for inclusion in International Journal of Aviation, Aeronautics, and Aerospace by an authorized administrator of Scholarly Commons. For more information, please contact commons@erau.edu. 


\section{Introduction}

In the aerospace industry, one of the most important subjects is guaranteeing the certification of an aircraft. All aircraft companies require that the quality of every component be accepted as ready to fly. This requirement makes it compulsory to inspect every component, specifically the fuselage, to detect potential defects before it is assembled into the aircraft. The complexity of the fuselage geometry makes it obligatory to have an automated system to inspect it using promising non-destructive testing (NDT) techniques. Many researchers have turned their attention to researching high frequency ultrasonic elastic waves. Elastic waves are capable of propagating over long distances in specimens, and tend to interact with changes such as cracks and delaminations in a workpiece (Wu \& Busse, 1998). Vibrothermography is based on ultrasonic waves as a NDT method in which cracks in an object are made visible through heating caused by high frequency ultrasounds wave. In this technique, the heat is generated through the dissipation of mechanical energy at the crack surfaces by ultrasonic waves. The frequency range used for excitation of structures is typically in the range 20 $\mathrm{kHz}$ to $100 \mathrm{kHz}$. The presence of the crack may result in a temperature rise around the damage area and the surface close to the crack. The temperature rise is measured by a high sensitivity infrared imaging camera whose field of view covers a large area. The method can cover large areas from a single excitation position so it is much faster than conventional ultrasonic testing or eddy current inspection, which requires scanning over the whole surface. In addition, vibrothermography can be a more convenient and reliable inspection technique for structures with complex geometries that are difficult to inspect by conventional methods (Gleiter, Riegert, Zweschper, \& Busse, 2006; Han, Islam, Newaz, Favro, \& Thomas, 2005). The technique is also particularly well-suited to the detection of cracks that can cause problems with other techniques such as conventional ultrasound and radiography. In fact, in this technique when waves are induced into the defects, mechanical energy will decay rapidly because of the friction between the crack surfaces. The elastic properties of the crack areas which are much more different than any other areas; consequently, thermoelastic and hysteresis effects are generated.

Finite element modeling (FEM) is useful technique to understand the effect of induced sound pulses on damaged zones and the principles behind crack detection using vibrothermography. FEM is also used to investigate effect of complex parameters, such as geometry, material properties, loads and nonlinearities (Han, Islam, Newaz, Favro, \& Thomas, 2006; Mabrouki, Genest, \& Thomas, 2009). FEM can be used to investigate heat generation which can be generated within the fuselage structure because of thermoelastic damping and friction effects. In this paper finite element modeling of elastic wave propagation 
was carried out in aluminum fuselage to understand how the waves propagate inside of the fuselage during vibrothermography technique. In this research, the fuselage was excited by dynamic loading at frequency of $20 \mathrm{KHz}$ and then influence of excitation on defect area was evaluated.

\section{Finite Element Modeling of Heat Generation in Cracks}

As previously mentioned, numerical approaches are useful to simulate ultrasonic wave propagation within specimen when the specimen under investigation has a complex geometry, as in many real-world applications. To simulate a UVT experiment, it is necessary to understand how mechanical stress waves, created by high frequencies above $20 \mathrm{KHz}$, propagate through material, and interact with a damaged area. The time step has to be small for both the implicit and explicit methods and small element length is required to solve the model accurately. In this paper, finite element analysis was conducted using COMSOL Multiphysics and ABAQUS commercial software. COMSOL as an implicit FEM solver has excellent capability to embed governing equations and design variables for modeling internal thermoelastic-viscoelastic heat generation. ABAQUS also has great built in features for modeling frictional heating at the contacting surfaces of a defect ("ABAQUS," n.d.; "COMSOL," n.d.). These basic heat sources and their constitutive equations are delivered in the following sections.

\section{Internal loss (Hysteresis heating)}

In the absence of plastic deformation, the internal heating sources of a structure subjected to high frequency vibration, can be classified as mechanical (viscoelastic) losses and nonlinear effects related to the thermoelastic damping.

Assuming Hooke's law is true, then if a cyclic vibrational stress is applied to a material, the resulting strain must be proportional and in phase with the stress. In reality, this does not happen and the amplitude of mechanical vibrations does decline with time until it is completely damped out. In other words, energy dissipation occurs in the material resulting in vibration attenuation. If a material is taken through one complete reversible stress cycle, an elastic hysteresis loop similar to the one shown in Figure 1a is formed. The amount of dissipated energy is the difference between the work done in straining the material and the work done by the elastic restoring forces. The delay phase angle $\delta$ between cyclic stress and strain is as represented in Figure $1 \mathrm{~b}$. 


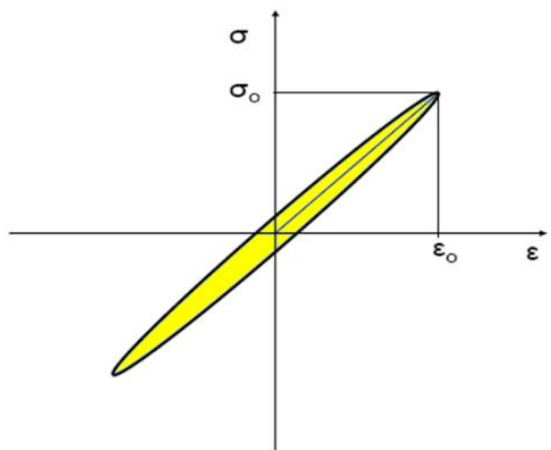

a)

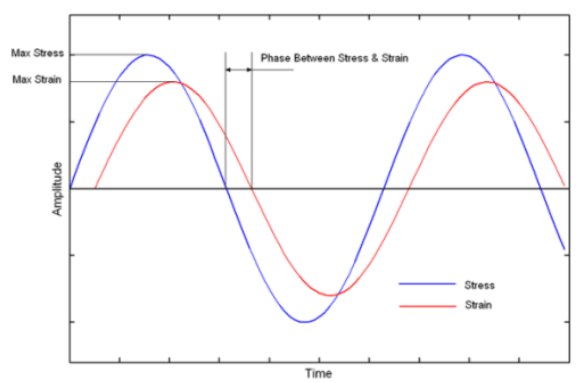

b)

Figure 1. Hysteresis damping and energy dissipation in a material. A) Hysteresis loop and dissipated energy; b) Delay phase angle between stress and strain.

Adapted from "FEM Modeling of Ultrasonic Vibrothermography of a Damaged Plate and Qualitative Study of Heating Mechanisms," by A. S. Rizi, S.

Hedayatrasa, X. Maldague, and T. Vukhanh, 2013, Journal of Infrared Physics \& Technology, 61, pp. 101-110.

Damping capacity or internal friction is a physical property that measures the ability of a material to convert mechanical energy of vibrations to heat which is dissipated through the volume of the material. Damping occurs in materials because of internal resistive forces that interact with cyclic stresses from mechanical vibrations and generally is due to the sum of several energy dissipative mechanisms such as point defect, dislocating, grain boundary, domain and thermoelastic damping. The type of interaction differs for the different classes of materials.

Thermoelastic damping describes energy dissipation arising from stress induced thermal gradients in a material. When a material is heated, the temperature rise causes dimensional changes. Likewise, when a material is stressed, the strain developed causes a rise in temperature. If the stress is 
homogeneous, the strain response is uniform and the temperature rise is even throughout. If the stress is inhomogeneous, local thermal gradients are developed in the materials which results in heat flow. Hence, thermoelastic damping is a main source of heating in defects because of stress inhomogeneity near crack area (Biot, 1956; Richard, \& Eslami, 2008).

To simulate the temperature rise in specimen due to internal hysteresis heat generation in ultrasonic loading, the following well-known conservation equation of energy is used:

$$
T \frac{\partial S}{\partial t}-\nabla(K \nabla T)=Q
$$

Where $S$ is entropy, $T$ the temperature averaged over a time period $2 \pi / \omega, Q$ heat generation rate $(\mathrm{W} / \mathrm{m} 3)$ and $k$ the thermal conductivity matrix $(W / m \cdot K)$. Accordingly, the temperature field is decomposed into small-amplitude periodic oscillations and slow temperature rise which are obtained from following equations respectively (Piau, Bendada, Maldague, \& Legoux, 2008):

$$
\begin{gathered}
\rho C_{P} \frac{\partial T}{\partial t}-\nabla(K \nabla T)=Q_{d}+Q_{2} \\
-\nabla\left(K \nabla T_{1}\right)=Q_{1}
\end{gathered}
$$

Where $\rho$ is density $\left(\mathrm{kg} / \mathrm{m}^{3}\right), C_{P}$ specific heat capacity $(\mathrm{J} / \mathrm{kg} . \mathrm{K}), T_{1}$ linear temperature response in the frequency domain and $Q_{1}, Q_{2}$ and $Q_{d}$ are:

$$
\begin{gathered}
Q_{1}=-j \omega\left(\rho C_{P} T_{1}+T S_{\text {elast }}\right) \\
Q_{2}=-\frac{1}{2} \operatorname{real}\left[T_{1} \operatorname{Conj}\left(j \omega S_{\text {elast }}\right)\right] \\
Q_{d}=\frac{1}{2} \omega \eta \cdot r e a l[\operatorname{Conj}(D . \varepsilon)]
\end{gathered}
$$

Where

$$
S_{\text {elast }}=\alpha D\left[\varepsilon-\alpha\left(T-T_{0}\right)\right]
$$

In which $j$ is the imaginary number, $D$ elasticity matrix, $\varepsilon$ strain vector, $\eta$ loss factor, $\alpha$ thermal expansion vector and $T_{0}$ the initial (strain reference) temperature. The term $Q_{d}$ presents the energy dissipation because of the inelastic (viscous) forces over the period, and $Q_{2}$ is the energy transfer between the mechanical and thermal domains due to the nonlinear nature of thermo elasticity.

\section{External loss (Frictional heating)}

In addition to internal losses, crack heating in UVT can be interpreted by frictional heating. In this context the temperature rise arises from rubbing friction of crack contact surfaces. Therefore, the coupling between thermal and mechanical problems takes place through the internal heat generation caused by 
the conversion of the frictional energy into thermal energy through an irreversible process ("COMSOL," n.d.). At the first step of our simulation, the contact pressure, calculated by finite element modeling, is used as a required parameter to stimulate the heat generation in cracks. In fact, from theoretical point of view, when the friction force $F$ moves through the distance $x$, an amount of energy is produced. The power input in friction is the product of the frictional force $F$ and the sliding velocity $v$. The input energy is balanced at the friction interface almost completely by heat dissipation away from the interface, either into the contacting solids or by radiation and convection to the surroundings. In general, around 5\% of the frictional energy is consumed or stored in the material as microstructural changes such as dislocations and phase transformation, or surface energy due to wear by rubbing of crack surfaces, etc. (Renshaw, Holland, \& Barnard, 2009). The remaining part of the frictional energy raises the interface temperature locally. In frictional heating, basically, the temperature rise at the interface is given as a function of the total heat developed, $q$ given by:

$$
q=\mu p v
$$

where $v$ is the sliding velocity, $\mu$ the friction coefficient and $p$ the pressure in the contact. The crack surfaces slip together when the structure is excited by ultrasonic waves and the amount of the contact pressure is proportional to many parameters such as static load applied by the horn, excitation frequency and material properties. In finite element modeling it is possible to express the equation 8 as follows ("ABAQUS," n.d.):

$$
Q(t)=\sum_{j=0}^{t / \delta t} \sum_{i=1}^{N}\left\{\left[\mu_{d}+\left(\mu_{s}-\mu_{d}\right) e^{-c\left|-v_{i}\left(t_{j}\right)\right|}\right] p_{i}\left(t_{j}\right) v_{i}\left(t_{j}\right) \delta A_{i}\right\} \delta t
$$

Equation 9 is summed over all $N$ nodes on the crack face and also over all time intervals, up to the time $t$ at which $Q(t)$ is calculated. Thus, $Q$ is a cumulative dissipation of energy over the entire crack face as a function of time. In Equation 9, $\mu_{s}$ and $\mu_{d}$ are the static and dynamic coefficients of friction at the crack, the constant $c$ is a number related to the velocity at which the transition from static to dynamic friction occurs, $v_{i}\left(t_{j}\right)$ the velocity of the $i^{t h}$ element at time $t j, P_{i}\left(t_{j}\right)$ the pressure between the $i^{\text {th }}$ element and its neighbor across the crack, and $\delta A_{i}$ the area of $i^{t h}$ element at the crack surface. Similarly, $\delta t$ is the time interval used in the computation, and $t_{j}=j \delta t$. 


\section{Modeling Procedure}

In our research, we model an aircraft fuselage with a very small crack near the window of aluminum fuselage sector as shown in Figure 2. The analysis for aluminum fuselage is performed using material properties presented in the Table 1 with different amplitudes and frequencies to observe the temperature rise in the crack area based on frictional heating.

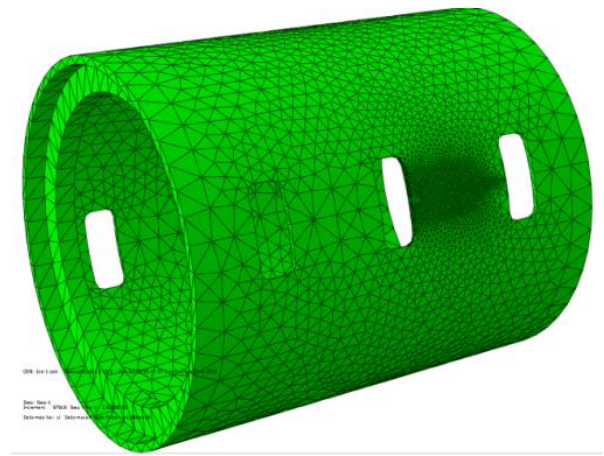

Figure 2. Geometry model for the FEM.

Table 1.

Material properties assumed for fuselage

\begin{tabular}{ccc}
\hline Item & Unit & Qty \\
\hline Young's Modulus E & $\mathrm{GPa}$ & 73 \\
Poisson Ratio $v$ & --- & 0.33 \\
Density $\rho$ & $\mathrm{Kg} /$ & 2780 \\
& $\mathrm{~m} 3$ & \\
Coefficient of & --- & 1.4 \\
friction $\mu$ & & \\
Yield stress $\sigma \mathrm{Y}$ & $\mathrm{MPa}$ & 345 \\
Loss factor $\eta$ & --- & 0.3 to 10 \\
& & $\times 10-5$ \\
Thermal conductivity & $\mathrm{W} / \mathrm{m}$ & 121 \\
$\mathrm{k}$ & $2 . \mathrm{oC}$ & \\
Specific heat & $\mathrm{J} / \mathrm{Kg}$ & 875 \\
capacity CP &. $\mathrm{oC}$ & \\
\hline
\end{tabular}


A time-dependent sinusoidal displacement load is applied in on a circle partition fuselage. The applied displacement had amplitude of $0.05 \mathrm{~mm}$ with a frequency of $20 \mathrm{kHz}$, and was applied for duration of $10 \mathrm{~ms}$. In all our models, the convection heat transfer coefficient was set at $10 \mathrm{~W} / \mathrm{m} 2 . \mathrm{k}$, on all sides of the modeled fuselage.

\section{Modeling Results}

Based on the modeling procedure mentioned above, the temperature distribution in a damaged fuselage under dynamic loading was investigated. In such case, the amount of temperature rise is proportional to the excitation frequency and material property and also to the applied loads. It is observed that the highest temperature increase is in the point of crack tip that is modeled by combining stress-strain analysis with the heat equations to calculate heat generated (see Figure 3).

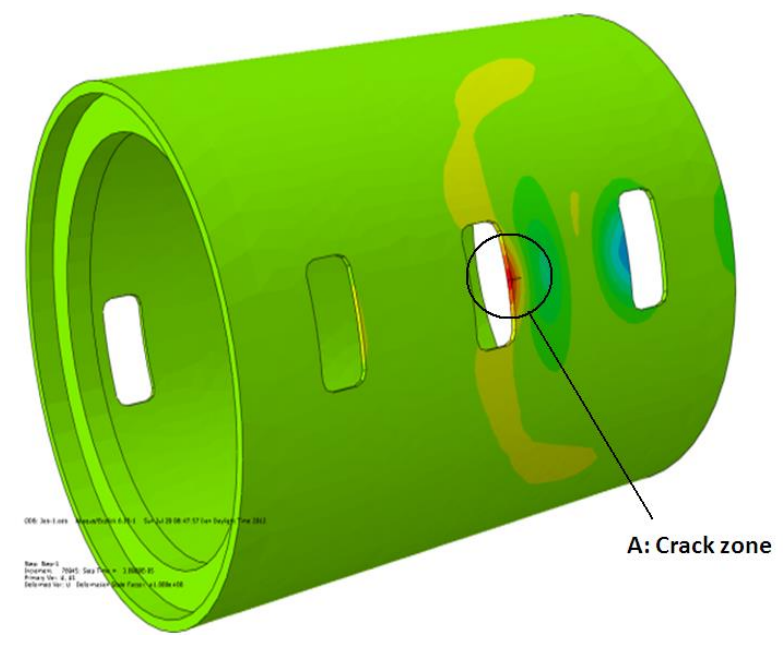

Figure. 3. Displacement distribution at fuselage subjected to $20 \mathrm{KHz}$ excitation load (Fuselage diameter: $3300 \mathrm{~mm}$, length of fuselage model: $4200 \mathrm{~mm}$ ).

The displacement and temperature distribution in defected fuselage subjected to $20 \mathrm{kHz}-0.05 \mathrm{~mm}$ excitation load are illustrated in Figure 4. 


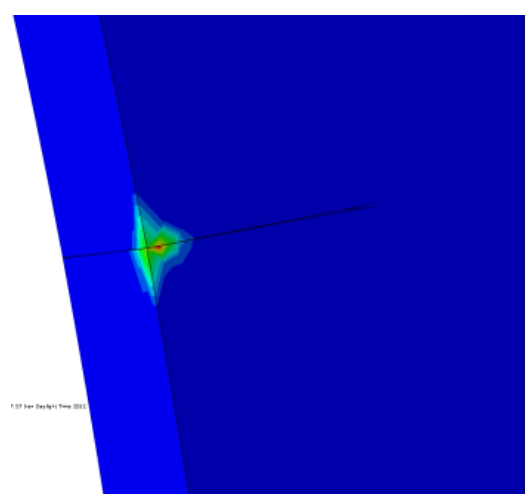

Figure 4. Temperature distribution in defect area of fuselage due to hysteresis heating (Magnification of zone A depicted in Figure 3).

It is clear that as the excitation time increases the temperature rise at the crack area in aluminum fuselage increases gradually with the exception that the temperature shows a low reduction which is probably due to wave energy dissipation (see Figure 5).

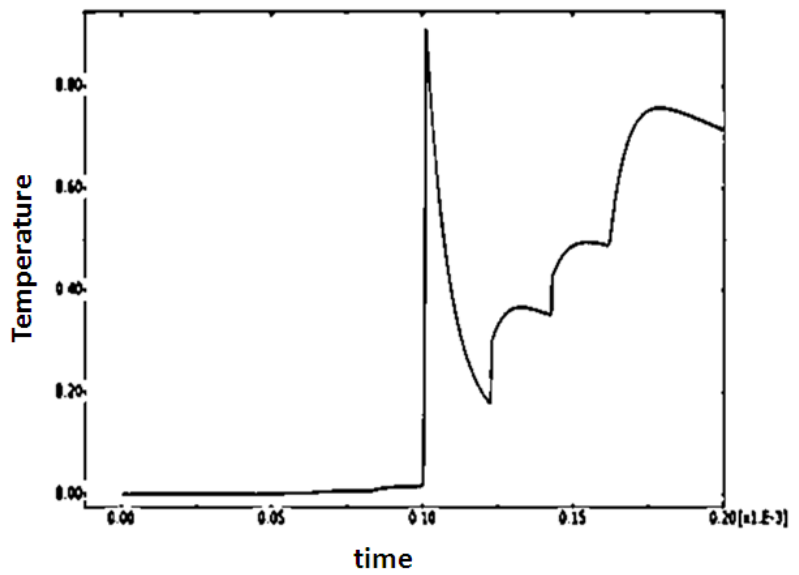

Figure 5. Temperature rise at edge crack tip versus time at $20 \mathrm{KHz}$ excitation load.

This delay occurs due to the reason that we are capturing the temperature rise at a node on the top of the upper plate and there is a distance between the data node and the heat source. It means that if the delamination was modeled at a location closer to the surface, this time delay would be decreased. Based on modeling results we observed that there is a limit for frequency and amplitude 
during vibrothermography such that dynamic loading above these limits can cause plastic deformation and also prevent the wave propagation inside the specimen. It should be noticed that plastic deformation in crack tip could cause a large temperature rise which is expected to have crack propagation and fatigue damage and nonlinear behavior in the frequency response. This point was considered during dynamic loading by high amplitude excitation during all steps of our modeling.

The heating patterns at crack area obtained from numerical results of this research are in good agreement with experimental UVT photos illustrated in Figures 6 (Renshaw, Holland, \& Barnard, 2009) and 7 (Rizi, Hedayatrasa, Maldague, \& Vukhanh, 2013; Xiaoyan, Zeng, Li, Islam, Lu, \& Loggins, 2004). Accordingly, the thermoelastic heating, which is proportional to stress gradients, is mostly generated at crack tips and no significant heat is generated in the length of crack.

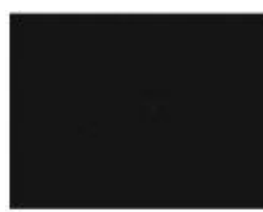

$60 \mathrm{~ms}$

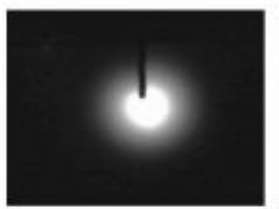

$450 \mathrm{~ms}$

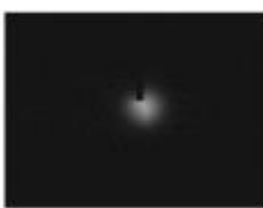

$150 \mathrm{~ms}$

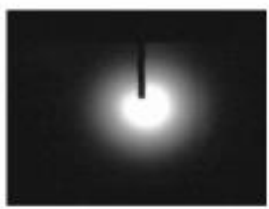

$600 \mathrm{~ms}$

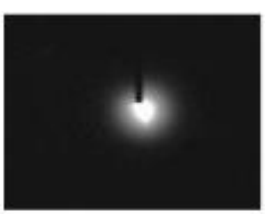

$300 \mathrm{~ms}$

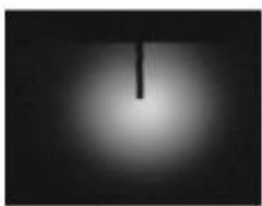

$750 \mathrm{~ms}$

Figure 6. Infrared images taken at different excitation stages in an Aluminum bar with $1 \mathrm{~mm}$ edge crack. Adapted from "Viscous Material-Filled Synthetic Defects for Vibrothermography," by J. Renshaw, S. D. Holland, and D. J. Barnard, 2009, NDT \& E International, 42(8), pp. 753-756. 


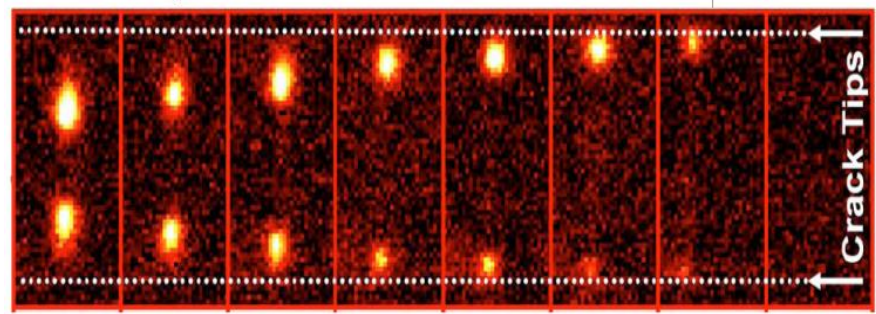

Figure 7. Infrared images of an internal crack. Adapted from "Acoustic Chaos for Enhanced Detectability of Cracks by Sonic Infrared Imaging," by H. Xiaoyan et al., 2004, Journal of Applied Physics, 95(7), pp. 3792-3797.

\section{Conclusion}

We developed a finite element method for damage heating in fuselage and learned how to obtain sufficient vibrations. The mechanism of friction and hysteresis and its relation with temperature rise was examined in small edge crack in aluminum fuselage. We studied these finite element analyses as the mechanism behind this technique to use it more effectively in fuselage inspection. At the next stage of our research, the integration and synchronization of the experimental vibrothermographic data along with FEM results will be used to design a fullyautomated system to inspect the whole aircraft fuselage. 


\section{References}

ABAQUS user manual version 6.5-1. (n.d.). Retrieved from https://classes.engineering.wustl.edu/2009/ spring/mase5513/abaqus/ docs/v6.5/index.html

Biot, M. A. (1956). Thermoelasticity and irreversible thermodynamics. Journal of Applied Physics, 27, 240-253. https://doi.org/10.1063/1.1722351

COMSOL multiphysics users guide -version 3.5. (n.d.). Retrevied from https://extras.csc.fi/ math/ comsol/3.4/doc/multiphysics/wwhelp/wwhimpl /js/html/wwhelp.htm?context=multiphysics\&file=html_modelingintro.15. 1.html

Gleiter, A., Riegert, G., Zweschper, T., \& Busse, G. (2006). Ultrasound lockin thermography for advanced depth resolved defect selective imaging. Paper presented at the European Conference on Nondestructive Testing, ECNDT Conference Proceedings, Berlin, Germany.

Han, X., Islam, M. S., Newaz, G., Favro, L. D., \& Thomas R. L. (2005). Finiteelement modeling of acoustic chaos to sonic infrared imaging. Journal of Applied Physics, 98(1), 21-32. https://doi.org/10.1063/1.1947382

Han, X., Islam, M., Newaz, G., Favro, L., \& Thomas, R. (2006). Finite element modelling of the heating of cracks during sonic infrared imaging. Journal of Applied Physics, 99(7), 074905-074905. https://doi.org/10.1063/1.2189023

Mabrouki, F., Genest, M., \& Thomas, A. (2009). Frictional heating model for efficient use of vibrothermography. NDT \& E International, 42(5), 345352.

Piau, J. M., Bendada, A., Maldague, X., \& Legoux, J. G. (2008). Nondestructive testing of open micro-cracks in thermally sprayed coatings using ultrasound excited vibrothermography." Nondestructive Testing and Evaluation, 23(2), 109-120.

Renshaw, J., Holland, S. D., \& Barnard, D. J. (2009). Viscous material-filled synthetic defects for vibrothermography. NDT \& E International, 42(8), 753-756.

Richard, B., \& Eslami, M. (2008). Thermal stresses: Advanced theory and applications. Berlin, Germany: Springer.

Rizi, A. S., Hedayatrasa, S., Maldague, X., \& Vukhanh, T. (2013). FEM modeling of ultrasonic vibrothermography of a damaged plate and qualitative study of heating mechanisms. Journal of Infrared Physics \& Technology, 61, 101-110. https://doi.org/10.1016/j.infrared.2013.07.011

Wu, D. T., \& Busse, G. (1998). Lock-in thermography for nondestructive evaluation of materials. Revue Generale De Thermique 37(8), 693-703.

Xiaoyan, H., Zeng, Z., Li, W., Islam, S., Lu, J. \& Loggins, V. (2004). Acoustic chaos for enhanced detectability of cracks by sonic infrared imaging. 
Journal of Applied Physics, 95(7), 3792-3797.

https://doi.org/10.1063/1.1652243 